\title{
Non-destructive analyses of defects and effects of airborne pollutants in historical buildings
}

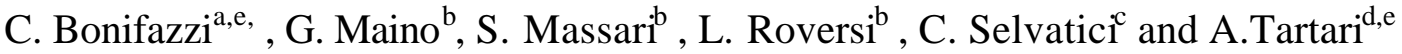 \\ ${ }^{a}$ Department of Biomedical Science, University of Ferrara - Italy \\ ${ }^{b}$ ENEA, Applied Physics Division, Bologna - Italy \\ ${ }^{c}$ DAPT, Alma Mater Studiorum, University of Bologna - Italy \\ ${ }^{d}$ Department of Physics, University of Ferrara-Italy \\ ${ }^{e}$ INFM, National Institute of Matter, Genova - Italy
}

Keywords. Material testing, Termography, ECoSp, PCA, Cultural Heritage Conservation.

Different imaging techniques are presented and applied to the study of historical buildings, mainly belonging to Renaissance time, in the city of Bologna, where the arenaria (sandstone) of which they are constituted is largely degradated in the surfaces due to the effects of pollutants present in the atmosphere. In the following figures, present status and a map od degradation effects is shown for two well-known palaces once belonging to honorable families, Bevilacqua and Fantuzzi (upper and lower figures).
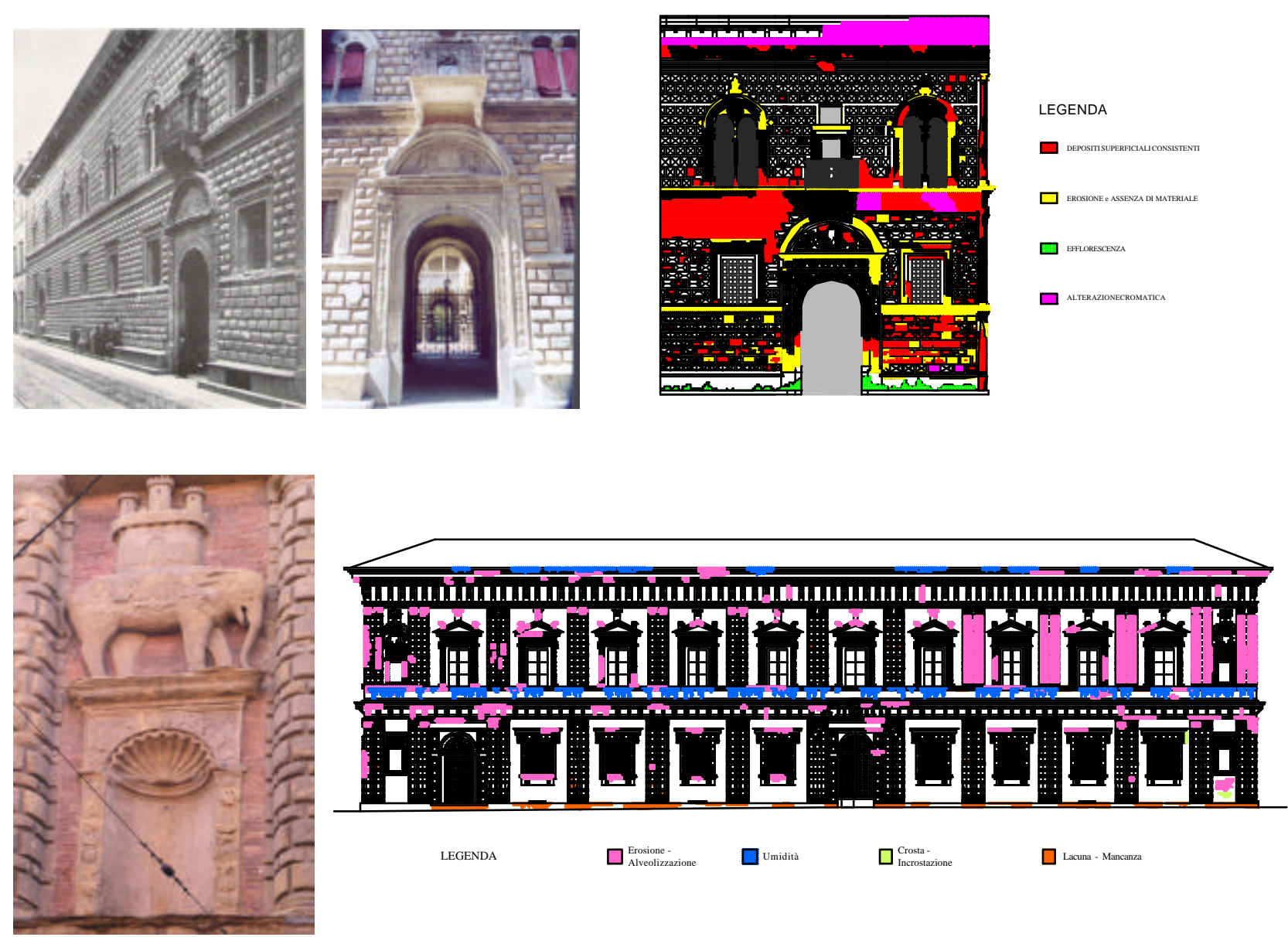
We based our investigation on multispectral techniques ranging from infrared reflectography, digitag termography and ultraviolet fluorescence. In addition, an original portable equipment based on the detection of Compton scattering photons and a further statistical analysis of the collected data has been utilized. The photon detection has been performed by the Enhanced Compton Spectrometer (ECoSp), a recently devised instrument that allows to collect the backscattered photons by investigating the tested sample from one side only. Photons collected during the planar scanning of a given area are used to describe the electronic density of the sample as a density image; afterwards, the Principal Component Analysis (PCA) was applied to the multispectral image processing, resulting from the mathematical combination of all the adopted imaging techniques. As a case study, a non-destructive testing of plaster substrates supporting mural paintings has been performed using this technique, searching for flaws, defects, fractures, and so on. Results are shown in the following figures in comparison with usual termographic analyses.
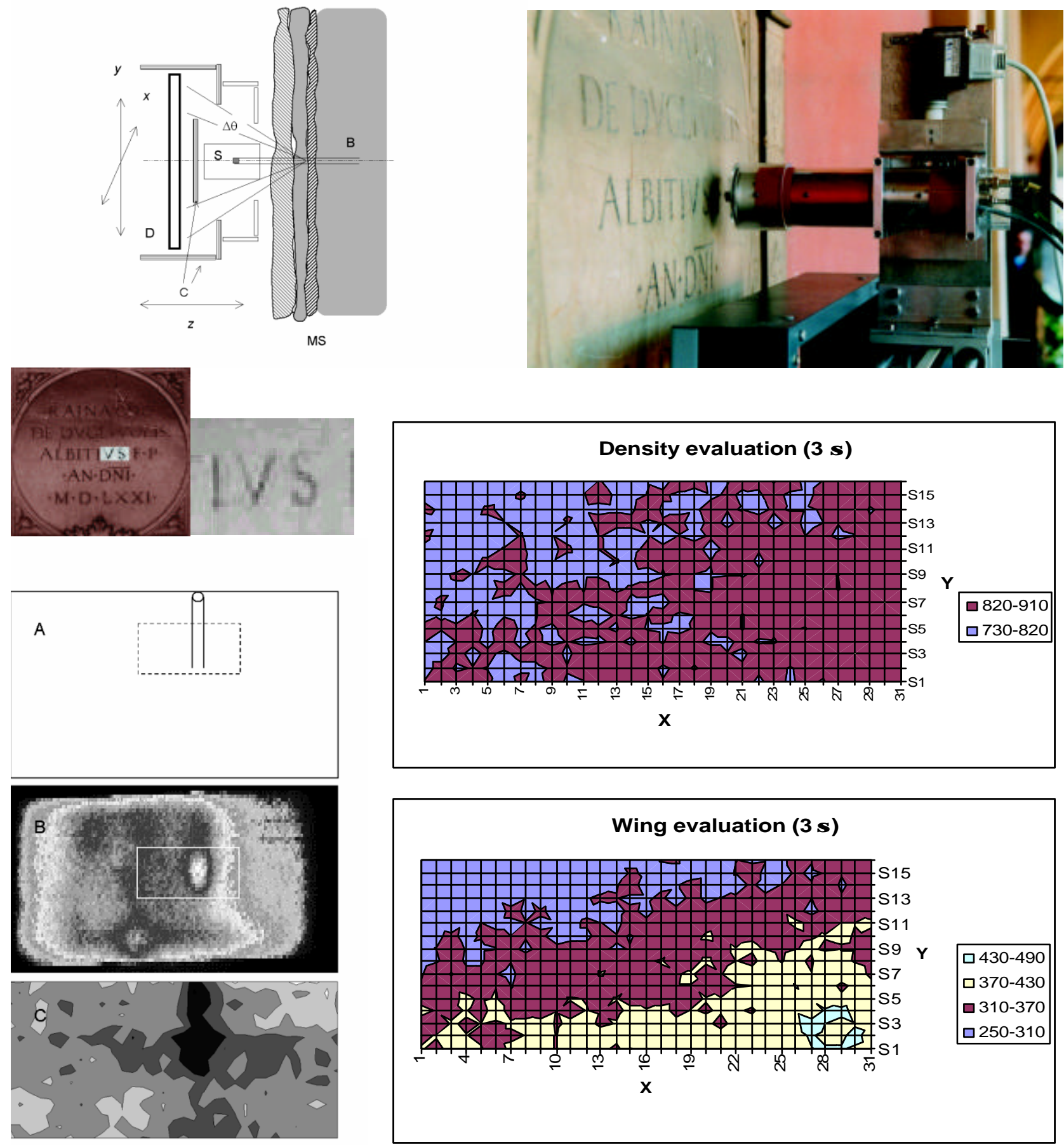